\title{
The Ionosphere Prediction Service
}

F. Rodriguez $\left({ }^{1}\right)$, L. R. Ronchini $\left({ }^{1}\right)$, S. Di $\operatorname{Rollo}\left({ }^{1}\right)$, G. De Franceschi $\left({ }^{2}\right)$, C. Cesaroni $\left({ }^{2}\right)$, L. Spogli $\left({ }^{2}\right)$, V. $\operatorname{Romano}\left({ }^{2}\right)$, M. Aquino $\left({ }^{3}\right)$, S. $\operatorname{Veettil}\left({ }^{3}\right)$, F. Berrilli $\left({ }^{4}\right)$, D. Del Moro $\left({ }^{4}\right)$, M. Hutchinson $\left({ }^{5}\right)$, O. Kalden $\left({ }^{6}\right)$,

A. Aragon-Angel $\left({ }^{7}\right)$ and E. Guyader $\left({ }^{8}\right)$

$\left({ }^{1}\right)$ TELESPAZIO - Rome, Italy

${ }^{2}$ ) Istituto Nazionale di Geofisica e Vulcanologia - Via di Vigna Murata 605, Rome, Italy

${ }^{3}$ ) University of Nottingham - Nottingham, UK

$\left({ }^{4}\right)$ University of Rome "Tor Vergata" - Rome, Italy

$\left({ }^{5}\right)$ Nottingham Scientific Limited - Nottingham, UK

$\left({ }^{6}\right)$ TELESPAZIO VEGA - Darmstadt, Germany

$\left({ }^{7}\right)$ Joint Research Centre, Directorate for Space, Security and Migration - Ispra, Italy

$\left.{ }^{8}\right)$ European Commission - Brussels, Belgium

received 28 December 2018

Summary. - The Ionosphere Prediction Service (IPS), project funded by European Commission within Horizon 2020 and currently ongoing, provides a prototype for a monitoring and prediction service of potential ionosphere-related disturbances affecting GNSS (Global Navigation Satellite System) user communities, to help these communities cope with the effects of the ionosphere and mitigate the related effects for the specific GNSS-based application/services. The aim of the IPS project is to design and develop a prototype platform able to translate the prediction and forecast of the ionosphere effects into a service customized for specific GNSS user communities. The objective is to alert the GNSS users in due time of an upcoming ionospheric event potentially harmful for GNSS and for the related operations in the given application field. The project team is composed of Telespazio (coordinator), Nottingham Scientific Ltd, Telespazio Vega DE, The University of Nottingham, The University of Rome Tor Vergata and the National Institute of Geophysics and Volcanology. The Joint Research Centre of the European Commission is also involved in the project.

\section{1. - Introduction}

The ionospheric fluctuations are the largest contributor to the error budget for GNSS receivers $[1,2]$. The principal Space-Weather related drivers to ionospheric fluctuations 
are solar flares and Coronal Mass Ejections (CMEs) [3,4]. Moreover, the inhomogeneity of ionospheric electron distribution (i.e. ionospheric irregularities) can cause sudden, rapid and irregular fluctuations of the amplitude and phase of the received signals [5]. Prediction and forecasting of the ionosphere state depend on our knowledge and understanding of the solar-terrestrial interactions $[6,7]$ and of the physics of the ionosphere [8]. IPS further translates the prediction and forecast of the ionosphere state into warning and predictions of performance degradation of GNSS systems.

The IPS development is conceived of two concurrent activities: the design and development of the prototype service and the research activity that will run along the whole project. The IPS products are fine-tuned to match the different needs of the communities (aviation, mass market, critical infrastructures monitoring etc.) to which the service is targeted. The design and development of the service is organized in four phases: the user requirements collection, the architecture specification, the implementation and validation of the prototype. A sub-activity analyses also the integration feasibility in the Galileo Service Centre, located in Madrid. The nowcasting and forecasting tools of IPS are based on the outputs of the research activity that represents the scientific backbone of IPS and it provides the models and algorithms for the forecasting products. The main products are related to Solar and Space-Weather Monitoring, Ionosphere weather monitoring and forecasting and receivers performance monitoring and they are complemented with specific aviation related receiver performance figures. The core of the IPS platform is the Central Processing and Storage Facility (CPSF) that manages the output of a chain of processors capable to describe and forecast the Space-Weather phenomena from the Sun to the Ionosphere affecting the receiver performance. The service is available through a web portal since July 2018 at the Internet address ips.gsc-europe.eu. In this paper, we describe the IPS project, the whole service chain and an overview of the available products.

\section{2. - The IPS Processing Chain}

The IPS prototype service is based on a complex architecture in which several elements are contributing to the overall processing chain, starting from the input data sources, the products processors, the central storage and ending with the user interface. Two are the main elements of the IPS architecture:

Remote Processing Facilities (RPFs): these processing elements represent the product generation elements that starting from Solar phenomena analysis and ionospheric products are able to predict the performance of the GNSS at user level. They interacts with both the remote sensors for the collection of the needed input data and the central storage to save the generated products, retrieve and process data from other RPFs or trigger one or more processes implemented in the central unit.

Central Storage and Processing Facility (CSPF): this is the IPS central facility that implements all the functionalities related to the collection and distribution of the products and the interactions with the GNSS user communities including notification, warnings and generation of periodic reports. The interaction with the users is possible through an innovative Web Portal that provides to the users the tool to access to the IPS products. The system is designed to operate in real time processing data coming from external sensors. This layer collects all the elements used to gather raw data for the space weather and ionospheric products generation. 


\section{3. - The IPS Products}

IPS provides 160 different products, that can be divided in several subclasses: 18 products about Solar physics related events (developed by the University of Tor Vergata), including observations and algorithms for flare nowcasting and forecasting $[9,10]$; nowcasting and long-term forecasting for CMEs released by the Sun [11,12]; measurement and forecasting of CME-related SEP fluxes [13].

63 products about Ionospheric phenomena related events (developed by the Istituto Nazionale di Geofisica e Vulcanologia), including the study of the morphology and dynamics of the ionospheric plasma to develop TEC; scintillation mapping and modelling on different temporal and spatial scales; nowcast, short-term and long-term forecast mapping tools on global and/or regional scale.

26 products about GNSS performance figures (developed by University of Nottingham), including modelling and prediction of GNSS tracking errors, loss of signal lock and expected levels of positioning errors; detection of Travelling Ionospheric Disturbances (TIDs) from Global TEC maps in collaboration with INGV.

37 products about GNSS service performance for aviation users (developed by Telespazio), including monitoring and forecasting of effects of ionosphere on the performance of GNSS systems at service level; ABAS forecast and SBAS nowcast services (accuracy, integrity, availability and continuity). 16 products about predefined statistics (developed by Telespazio).

All IPS Products are available to the final user in the form of Widgets, in particular: global and regional scale products can be displayed in the form of jpg images in the web portal and also in the form of maps widgets where the user can pan and zoom; other time-series type products can be displayed as plots where the user can select the time interval, and some products producing large output data can be displayed as tables widgets; finally, those IPS products consisting into scalar values can be represented by gauges, and they can also be used to set active alarms.

The authors acknowledge the funding from the EC through the IPS project [contract number: 434/PP/GRO/RCH/15/8381].

\section{REFERENCES}

[1] AQUINO M. and SREEJA V., Space Weather, 11-5 (2013) 219.

[2] PARK J. et al., Radio Sci., 51 (2016) 429

[3] NARICI L. et al.,, in Extreme Events in Geospace, edited by BUZULUKOVA N. (Elsevier, Amsterdam) 2018.

[4] PIERSANTI M. et al., Solar Phys., 292 (2017) 169.

[5] SPOGLI L. et al., J. Atmos. Solar-Terr. Phys., 105 (2013) 199

[6] BERRILLI F. et al., J. Space Weather Space Clim., 4 (2014) A16.

[7] DI FINO L. et al., J. Space Weather Space Clim., 4 (2014) A19.

[8] CeSAROni C. et al., J. Geophys. Res. A: Space Phys., 122 (2017) 11794.

[9] FORTE R. et al.,, in Space Weather of the Heliosphere: Processes and Forecasts, edited by FOULLON C. and MALANDRAKI O., Vol. 335 (Cambridge University Press, London) 2018. 
[10] SCHRIJVER C. J., Astrophys. J. Lett., 655 (2007) L117.

[11] NAPOLETANO G. et al., J. Space Weather Space Clim., 8 (2018) A11.

[12] NAPOlEtano G. et al.,, in Space Weather of the Heliosphere: Processes and Forecasts, edited by FOULLON C. and MALANDRAKI O., Vol. 335 (Cambridge University Press, London) 2018.

[13] PAPAIOANnOU A. et al., J. Space Weather Space Clim., 6 (2016) A42. 